\title{
Obstructive sleep apnoea during REM sleep and incident non-dipping of nocturnal blood pressure: a longitudinal analysis of the Wisconsin Sleep Cohort
}

\author{
Babak Mokhlesi, ${ }^{1}$ Erika W Hagen, ${ }^{2}$ Laurel A Finn, ${ }^{2}$ Khin Mae Hla, ${ }^{3}$ Jason R Carter, ${ }^{4}$ \\ Paul E Peppard ${ }^{2}$
}

- Additional material is

published online only. To view please visit the journal online (http://dx.doi.org/10.1136/ thoraxjnl-2015-207231).

For numbered affiliations see end of article.

\section{Correspondence to} Dr Babak Mokhlesi, Section of Pulmonary and Critical Care Medicine, Sleep Disorders Center, University of Chicago, 5841 S. Maryland Ave, MC6076/Room M630, Chicago, IL 60637, USA; bmokhles@medicine.bsd. uchicago.edu

Received 27 April 2015 Revised 20 July 2015

Accepted 11 August 2015 Published Online First 25 August 2015

\section{CrossMark}

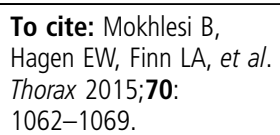

\section{ABSTRACT \\ Background Non-dipping of nocturnal blood pressure (BP) is associated with target organ damage and cardiovascular disease. Obstructive sleep apnoea (OSA) is associated with incident non-dipping. However, the relationship between disordered breathing during rapid eye movement (REM) sleep and the risk of developing non-dipping has not been examined. This study investigates whether OSA during REM sleep is associated with incident non-dipping. \\ Methods Our sample included 269 adults enrolled in the Wisconsin Sleep Cohort Study who completed two or more $24 \mathrm{~h}$ ambulatory BP studies over an average of 6.6 years of follow-up. After excluding participants with prevalent non-dipping BP or antihypertensive use at baseline, there were 199 and 215 participants available for longitudinal analysis of systolic and diastolic non- dipping, respectively. OSA in REM and non-REM sleep were defined by apnoea hypopnoea index (AHI) from baseline in-laboratory polysomnograms. Systolic and diastolic non-dipping were defined by systolic and diastolic sleep/wake BP ratios $>0.9$. Modified Poisson \\ Key messages \\ What is the key question? \\ - Does obstructive sleep apnoea (OSA) during rapid eye movement (REM) sleep increase the risk of developing non-dipping of nocturnal blood pressure? \\ What is the bottom line? \\ - OSA in REM sleep is longitudinally associated with incident non-dipping of systolic and diastolic blood pressure. \\ Why read on? \\ - Since REM sleep predominates in the early morning hours before typical awakening, the cardiovascular benefits of continuous positive airway pressure (CPAP) therapy may not be achieved with the typical CPAP use of 3-4 $\mathrm{h}$ per night.} regression models estimated the relative risks for the relationship between REM AHI and incident non-dipping, adjusting for non-REM AHI and other covariates.

Results There was a dose-response greater risk of developing systolic and diastolic non-dipping BP with greater severity of OSA in REM sleep ( $p$-trend $=0.021$ for systolic and 0.024 for diastolic non-dipping). Relative to those with REM AHI<1 event/h, those with REM $\mathrm{AHI} \geq 15$ had higher relative risk of incident systolic nondipping $(2.84,95 \% \mathrm{Cl} 1.10$ to 7.29$)$ and incident diastolic non-dipping $(4.27,95 \% \mathrm{Cl} 1.20$ to 15.13$)$. Conclusions Our findings indicate that in a population-based sample, REM OSA is independently associated with incident non-dipping of BP.

\section{INTRODUCTION}

Blood pressure (BP) follows a diurnal pattern, increasing during the day and dipping during nocturnal sleep. Ambulatory BP monitoring (ABPM) can measure night-day patterns of BP over a $24 \mathrm{~h}$ period and provides more useful prognostic information than clinic measurements of $\mathrm{BP}^{1-3}$ In healthy individuals BP normally varies during different physiologic states and declines by more than $10-20 \%$ at night-time during sleep compared with daytime waking $\mathrm{BP}^{4}$ Non-dipping is traditionally defined as nocturnal BP decrease less than $10 \%$ of daytime BP. ${ }^{2}$ Non-dipping has important clinical implications because it is a marker for future development of hypertension in those who are normotensive and, in patients with hypertension, nocturnal hypertension and non-dipping have been associated with worse cardiovascular prognosis and increased target organ damage, including left ventricular hypertrophy, myocardial infarction, angina, ischaemic stroke and cardiovascular death. ${ }^{56}$

Obstructive sleep apnoea (OSA), a highly prevalent chronic condition, ${ }^{7}$ has been independently associated with cardiovascular morbidity and mortality, ${ }^{8-10}$ including non-dipping nocturnal $\mathrm{BP}^{11}{ }^{12}$ Increased sympathetic activity is widely considered to be a major putative mechanism by which OSA increases cardiovascular risk. ${ }^{13} 14$ It is well established that compared with non-rapid eye movement (NREM) sleep, rapid eye movement (REM) sleep is associated with greater sympathetic activity and cardiovascular instability in healthy humans and in patients with OSA. ${ }^{13}{ }^{15}$ Therefore, OSA during REM sleep may have more serious adverse consequences than OSA during NREM sleep. Indeed, obstructive apnoeas and hypopneas during REM 
sleep are longer in duration and are associated with significantly greater oxygen desaturation compared with events in NREM sleep. ${ }^{16}{ }^{17}$ We recently reported that OSA during REM sleep was cross-sectionally and longitudinally associated with hypertension in the Wisconsin Sleep Cohort. ${ }^{18}$ The higher relative odds of prevalent and incident hypertension was most evident when the apnoea hypopnoea index (AHI) during REM sleep reached 15 events per hour. The longitudinal association between REM-related respiratory events and the risk of developing incident non-dipping of nocturnal BP remains largely unexplored. In community-based studies the majority of participants with $\mathrm{REM} A H I \geq 15$ would be clinically classified as having no OSA (overall AHI $<5$ ) or mild OSA (overall AHI 5-14.9). ${ }^{18} 19$ Although in clinical practice those with mild OSA are deemed at a low risk of future cardiovascular disease, it is important to ascertain whether this low risk also applies to individuals with an overall low AHI but significant OSA during REM sleep (ie, REM AHI $\geq 15$ ).

To that end, the objective of this prospective study was to determine the longitudinal association between REM AHI and the risk of developing non-dipping nocturnal BP. To address our objective, we performed longitudinal analysis of $24 \mathrm{~h}$ ambulatory BP recordings on a sample of Wisconsin Sleep Cohort Study (WSCS) participants, who were nocturnal BP dippers at baseline, to investigate whether OSA in REM sleep is longitudinally associated with incident nocturnal non-dipping over an average 6.6 years of follow-up.

\section{METHODS}

\section{Study sample}

The University of Wisconsin Health Sciences Institutional Review Board approved the WSCS protocols and informed consent documents. Details of the WSCS are provided in the online supplementary material. Since its inception in 1989 the Cohort has provided the opportunity for adding ancillary studies. In this study, a protocol for $24 \mathrm{~h}$ ambulatory BP was added to the protocol in 1991 with sequential enrolment. A total of 817 participants had at least one baseline ambulatory BP study. In our analysis we included subjects who had a minimum of $30 \mathrm{~min}$ of REM sleep on the baseline polysomnogram to ensure meaningful assessments of OSA during REM sleep and at least two ambulatory BP studies. ${ }^{20}$ We excluded subjects who used CPAP during the laboratory polysomnogram. We also excluded participants who were on antihypertensive medications or were non-dippers at their baseline ABPM study (assessed separately for systolic and diastolic analyses) to create an inception cohort, free of the condition of nocturnal BP nondipping, to follow for the development of new non-dipping (figure 1). Subjects who were hypertensive at baseline but were not on any antihypertensive medications and exhibited a normal dipping pattern at baseline were included in the study.

\section{Polysomnography}

Details of the in-laboratory polysomnography technique are provided in the online supplementary material. ${ }^{7}{ }^{21}$ REM AHI categories were examined as the independent variable: REM $\mathrm{AHI}<1$ (reference category), 1-4.9, 5-14.9, $\geq 15$.

\section{Ambulatory BP recordings}

Typically, the baseline $24 \mathrm{~h} \mathrm{ABPM}$ was performed within 6 weeks following the overnight sleep study, with the Accutracker II (SuntechMedical Instruments/Eutectics Electronics, Raleigh, North Carolina, USA), a $24 \mathrm{~h}$ BP monitoring device that uses a modified auscultatory method. ABPM readings were obtained at random intervals averaging every $15-20$ min during wakefulness and every $30 \mathrm{~min}$ during sleep. Activity, posture, bedtime, and time on awakening from sleep were recorded by participants on diaries. ABPM data were edited using predetermined established criteria. ${ }^{22}$ Individual mean BPs were computed by averaging ABPM measurements during sleep and wake defined by participants' recorded sleep and wake times, not by preset night-time and daytime cutoffs.

Systolic BP nocturnal non-dipping, assessed from ABPM, was defined as mean systolic sleep $\mathrm{BP} /$ mean systolic wake $\mathrm{BP}$ ratio $>0.9$. Diastolic non-dipping was defined analogously. An outcome variable was created to represent maintenance of dipping status (no development of non-dipping over observed follow-up) or incident non-dipping (development of nondipping during observed follow-up).

\section{Covariates}

Covariates included in the final models included age, sex, and race/ethnicity; clinically measured body mass index (BMI) and waist-to-hip ratio; and questionnaire-assessed smoking status and alcohol intake. For models exploring REM AHI categories, we also adjusted for NREM AHI. For models exploring the association between NREM AHI categories and incident nondipping we also adjusted for REM AHI. Further detail on covariates is provided in the online supplementary material.

\section{Statistical analysis}

All data were analysed with SAS software, V.9.3 (SAS Institute Inc, Cary, North Carolina, USA) and two-sided $\mathrm{p}$ values of $<0.05$ were considered to indicate statistical significance. Longitudinal change in nocturnal BP dipping status was modelled using modified Poisson regression to estimate relative risks and $95 \%$ CIs for the relationship between REM AHI severity categories and incident non-dipping over an average of 6.6 years follow-up, for systolic non-dipping and diastolic non-dipping separately. ${ }^{23}$ We chose to use modified Poisson regression model given that in our cohort the specific time of onset of the binary outcome (ie, onset of non-dipping) was unknown but was known to occur in a broad follow-up interval. Thus we assess risks (probabilities) of outcome, but not rates. The sample for this analysis included those who experienced normal nocturnal dipping (sleep/wake $\mathrm{BP}<0.9$ ) and were not taking antihypertensive medications at baseline (thus defined, 199 subjects were available to be followed for incident systolic non-dipping and 215 for incident diastolic non-dipping). These models included REM AHI categories: REM AHI $<1$ (reference category), 1-4.9, $5-14.9, \geq 15$ and were adjusted for $\log _{2}$ (NREM AHI +1$)$, age, sex, race/ethnicity, BMI, waist-to-hip ratio, smoking status, and alcohol use based on data from baseline visit. $\log _{2}$ (NREM AHI +1 ) allows for the coefficients to be interpreted as the 'effect' of a twofold increase in NREM AHI (1 was added to NREM AHI in the argument of the logarithm to allow for analysis of zero values). We also constructed models in which individual measures of sleep quality or duration were added to the abovementioned covariates one at a time to assess the impact on the association between REM AHI categories and incident nondipping. These covariates included self-reported habitual sleep time or several variables from the baseline polysomnogram, such as sleep efficiency, wake after sleep onset (WASO) and minutes of slow wave sleep (stage NREM 3). We also explored whether BMI at follow-up, presence of excessive daytime sleepiness, having been started on an antihypertensive medication, or having received treatment for OSA (ie, continuous positive airway pressure (CPAP)) had any impact on the relationship 


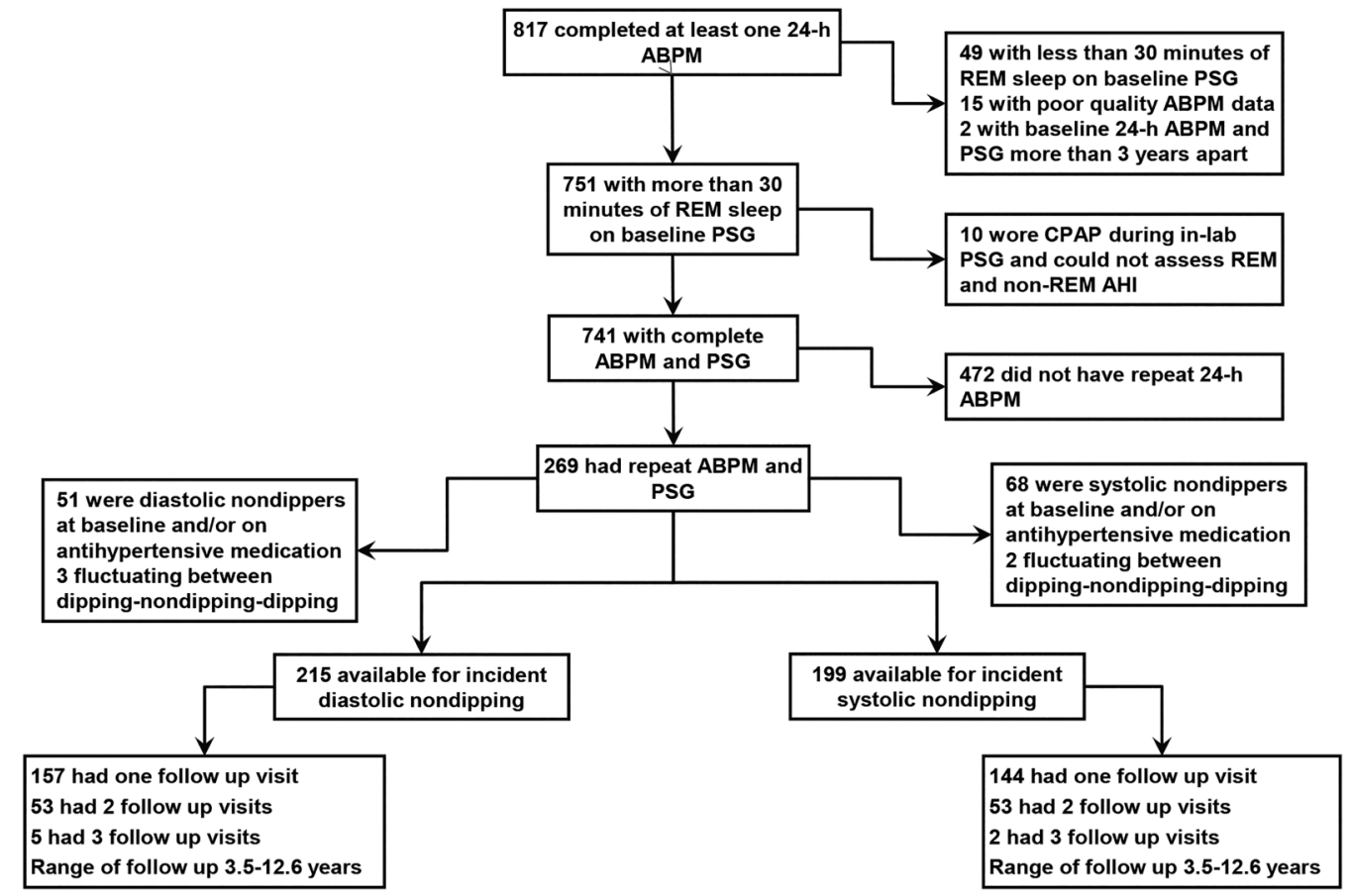

Figure 1 Flow chart of the study. ABPM, ambulatory blood pressure monitoring; AHI, apnoea hypopnoea index; CPAP, continuous positive airway pressure; PSG, polysomnogram; REM, rapid eye movement.

between REM AHI categories and incident non-dipping. Similar analyses were performed using total AHI categories: $\mathrm{AHI}<1$ (reference category), 1-4.9, 5-14.9, $\geq 15$ and NREM AHI categories: NREM AHI $<1$ (reference category), 1-4.9, 5-14.9, $\geq 15$. In the NREM AHI model we also adjusted for $\log _{2}(\mathrm{REM}$ $\mathrm{AHI}+1)$.

\section{RESULTS}

The flow of subjects included in the analytic sample is illustrated in figure 1. In table 1 we compare the baseline characteristics of the cohort who had $\geq 30$ min of REM sleep during the baseline polysomnogram and underwent at least two measurements of

Table 1 Comparison of baseline characteristics of participants who had repeat $24 \mathrm{~h}$ ABPM studies and follow-up polysomnogram (PSG) $(n=269)$ versus those participants with just one $24 \mathrm{~h}$ ABPM study $(n=741)$

\begin{tabular}{|c|c|c|}
\hline & $\begin{array}{l}\text { With follow-up ABPM } \\
\text { data }(n=269)\end{array}$ & $\begin{array}{l}\text { Only one ABPM } \\
\text { recording }(n=741)\end{array}$ \\
\hline Male, \% & 62 & 59 \\
\hline Age, years, mean (SD) & $49(8)$ & $50(8)$ \\
\hline $\begin{array}{l}\text { Body mass index, } \mathrm{kg} / \mathrm{m}^{2} \text {, } \\
\text { mean (SD) }\end{array}$ & $29(5)$ & $30(6)$ \\
\hline Race/ethnicity, white, \% & 97 & 94 \\
\hline Current smoker, \% & 17 & 19 \\
\hline $\begin{array}{l}\text { Alcohol, number of drinks/ } \\
\text { week (SD) }\end{array}$ & $4(5)$ & $4(6)$ \\
\hline Prevalent hypertension, \% & 30 & 35 \\
\hline $\begin{array}{l}\text { Antihypertensive } \\
\text { medication users, \% }\end{array}$ & 13 & 16 \\
\hline Type 2 diabetes, \% & 2 & 4 \\
\hline $\mathrm{AHI}$, mean (SD) & $5(9)$ & $5(10)$ \\
\hline
\end{tabular}

ABPM ( $n=269)$ versus all subjects who had $\geq 30$ min of REM sleep during the baseline polysomnogram and had at least one measurement of ABPM $(n=741)$. There were no systematic differences between the two groups. The baseline characteristics of the analytic cohort are summarised in table 2 (systolic nondipping) and table 3 (diastolic non-dipping). In both groups, the mean age was 48 years, mean BMI was $28 \mathrm{~kg} / \mathrm{m}^{2}, 59-60 \%$ were men, and $98 \%$ were white. The mean scores on the Epworth Sleepiness Scale was 9. The mean total sleep time was $390 \mathrm{~min}$ and REM sleep represented $19 \%$ of total sleep time. As expected, there was an increase in total AHI and NREM AHI across increasing REM AHI severity categories. Participants in the highest REM AHI category had a higher BMI, a higher prevalence of hypertension, and were more likely to be men.

During the follow-up period, 34 subjects (17\%) developed systolic non-dipping and 20 subjects (9\%) developed diastolic non-dipping. REM AHI severity at baseline was significantly associated with the onset of non-dipping systolic and diastolic BP. Table 4 summarises the adjusted relative risks for incident systolic and diastolic non-dipping based on REM AHI categories. After adjusting for age, sex, race, BMI, waist-to-hip ratio, smoking status, alcohol intake, and NREM AHI, there was a dose-response increased risk of developing systolic and diastolic non-dipping with increasing severity of OSA in REM sleep (p-trend 0.021 for systolic and 0.024 for diastolic non-dipping) (figure $2 \mathrm{~A}, \mathrm{~B}$ ). Those with $\mathrm{REM}$ AHI $\geq 15$ had higher relative risk of incident systolic non-dipping BP $(2.84,95 \%$ CI 1.10 to 7.29) and incident diastolic non-dipping (4.27, 95\% CI 1.20 to 15.13) than subjects with REM AHI $<1$. We also assessed the relative risk of incident non-dipping by NREM AHI categories and total AHI categories (figure 2). After adjusting for REM AHI and other covariates, higher NREM AHI categories were not associated with incident systolic or diastolic non-dipping (figure 2C, D). In contrast, higher total AHI categories were independently associated with systolic non-dipping, but not diastolic non-dipping (figure 2E, F). 
Table 2 Sample characteristics by REM AHI severity category for incident systolic non-dipping $(n=199)$

\begin{tabular}{|c|c|c|c|c|c|}
\hline & \multicolumn{4}{|c|}{ REM AHI severity category } & \multirow[t]{2}{*}{ Total } \\
\hline & $<1$ & $1-4.9$ & $5-14.9$ & $\geq 15$ & \\
\hline $\mathrm{N}$ & 73 & 60 & 38 & 28 & 199 \\
\hline Male, \% & 47 & 63 & 66 & 82 & 60 \\
\hline Age, years, mean (SD) & $46(8)$ & $49(7)$ & $50(7)$ & $49(9)$ & $48(8)$ \\
\hline Body mass index, $\mathrm{kg} / \mathrm{m}^{2}$, mean (SD) & $27(4)$ & $27(4)$ & $30(4)$ & $31(5)$ & $28(4)$ \\
\hline Race/ethnicity, white, \% & 99 & 98 & 95 & 100 & 98 \\
\hline Current smoking, \% & 16 & 17 & 18 & 21 & 17 \\
\hline Alcohol, number of drinks/week (SD) & $4(5)$ & $3(5)$ & $5(6)$ & $5(6)$ & $4(6)$ \\
\hline Prevalent hypertension, $\%$ & 4 & 23 & 13 & 57 & 19 \\
\hline Type 2 diabetes, $\%$ & 0 & 0 & 0 & 0 & 0 \\
\hline Waist-to-hip ratio, mean (SD) & $0.9(0.1)$ & $0.9(0.1)$ & $0.9(0.1)$ & $1.0(0.1)$ & $0.9(0.1)$ \\
\hline AHI, mean (SD) & $0.6(1)$ & $3(5)$ & $4(3)$ & $18(19)$ & $4(9)$ \\
\hline NREM AHI, mean (SD) & $0.7(1)$ & $3(6)$ & $3(3)$ & $15(20)$ & $4(9)$ \\
\hline REM AHI, mean (SD) & $0.2(0.3)$ & $3(1)$ & $8(2)$ & $30(13)$ & $7(11)$ \\
\hline Minutes total sleep time, mean (SD) & $393(55)$ & $393(54)$ & $371(69)$ & $404(66)$ & $391(60)$ \\
\hline Minutes REM sleep, mean (SD) & $76(27)$ & $77(28)$ & $70(25)$ & $66(25)$ & $74(27)$ \\
\hline Percent REM sleep, mean (SD) & $19(6)$ & $19(6)$ & $19(5)$ & $16(5)$ & $19(6)$ \\
\hline Minutes slow wave sleep, mean (SD) & $43(29)$ & $45(37)$ & $37(31)$ & $36(35)$ & $41(33)$ \\
\hline Percent slow wave sleep, mean (SD) & $11(7)$ & $12(9)$ & $9(7)$ & $9(9)$ & $11(8)$ \\
\hline Epworth Sleepiness Scale*, mean (SD) & $9(4)$ & $9(4)$ & $9(3)$ & $12(4)$ & $9(4)$ \\
\hline Excessive daytime sleepiness, \% & 26 & 12 & 29 & 21 & 22 \\
\hline
\end{tabular}

${ }^{*}$ Only available on a subset of studies $(n=110)$.

AHI, apnoea hypopnoea index; NREM, non-rapid eye movement; REM, rapid eye movement.

Results were essentially unchanged in models that also adjusted for BMI at follow-up or whether an individual was initiated on antihypertensive medication during the follow-up time period. We also explored whether initiation of home CPAP therapy could alter this relationship. Only four subjects in the systolic group and five subjects in the diastolic group were initiated on home CPAP therapy during the course of the study. Models that also adjusted for home CPAP therapy were essentially unchanged. Moreover, models that adjusted for various measures of sleep, such as self-reported habitual sleep duration,

Table 3 Sample characteristics by REM AHI severity category for incident diastolic non-dipping ( $n=215)$

\begin{tabular}{|c|c|c|c|c|c|}
\hline & \multicolumn{4}{|c|}{ REM AHI severity category } & \multirow[t]{2}{*}{ Total } \\
\hline & $<1$ & $1-4.9$ & $5-14.9$ & $\geq 15$ & \\
\hline $\mathrm{N}$ & 83 & 61 & 40 & 31 & 215 \\
\hline Male, $\%$ & 48 & 61 & 63 & 77 & 59 \\
\hline Age, years, mean (SD) & $46(8)$ & $49(7)$ & $51(7)$ & $49(9)$ & $48(8)$ \\
\hline Body mass index, $\mathrm{kg} / \mathrm{m}^{2}$, mean (SD) & $27(4)$ & $28(4)$ & $29(4)$ & $32(5)$ & $28(5)$ \\
\hline Race/ethnicity, white, \% & 99 & 98 & 95 & 100 & 98 \\
\hline Current smoking, \% & 16 & 16 & 20 & 23 & 18 \\
\hline Alcohol, number of drinks/week, (SD) & $3(5)$ & $3(4)$ & $5(6)$ & $5(6)$ & $4(5)$ \\
\hline Prevalent hypertension, \% & 7 & 20 & 15 & 52 & 19 \\
\hline Type 2 diabetes, \% & 0 & 0 & 0 & 0 & 0 \\
\hline Waist-to-hip ratio, mean (SD) & $0.9(0.1)$ & $0.9(0.1)$ & $0.9(0.1)$ & $0.9(0.1)$ & $0.9(0.1)$ \\
\hline $\mathrm{AHI}$, mean $(\mathrm{SD})$ & $0.9(3)$ & $3(5)$ & $4(3)$ & $17(17)$ & $4(9)$ \\
\hline NREM AHI, mean (SD) & $1(3)$ & $3(6)$ & $3(3)$ & $14(19)$ & $4(9)$ \\
\hline REM AHI, mean (SD) & $0.2(0.3)$ & $2(1)$ & $8(3)$ & $30(13)$ & $7(11)$ \\
\hline Minutes total sleep time, mean (SD) & $390(54)$ & $392(54)$ & $373(66)$ & $399(68)$ & $389(59)$ \\
\hline Minutes REM sleep, mean (SD) & $75(27)$ & $79(29)$ & $70(26)$ & $65(25)$ & $74(27)$ \\
\hline Percent REM sleep, mean (SD) & $19(6)$ & $20(6)$ & $19(6)$ & $16(5)$ & $19(6)$ \\
\hline Minutes slow wave sleep, mean (SD) & $42(30)$ & $45(36)$ & $39(32)$ & $33(35)$ & $41(33)$ \\
\hline Percent slow wave sleep, mean (SD) & $11(8)$ & $10(8)$ & $10(8)$ & $8(9)$ & $11(8)$ \\
\hline Epworth Sleepiness Scale*, mean (SD) & $9(4)$ & $9(4)$ & $9(4)$ & $11(5)$ & $9(4)$ \\
\hline Excessive daytime sleepiness, \% & 24 & 10 & 33 & 19 & 21 \\
\hline
\end{tabular}

${ }^{*}$ Only available on a subset of studies ( $\left.n=117\right)$.

AHI, apnoea hypopnoea index; NREM, non-rapid eye movement; REM, rapid eye movement. 
Table 4 The association of REM AHI severity category with incident* systolic and diastolic non-dipping over an average of 6.6 years of follow-up using modified Poisson regression

\begin{tabular}{llll}
\hline $\begin{array}{l}\text { REM AHI } \\
\text { category }\end{array}$ & $\begin{array}{l}\text { Total } \\
\text { sample } \\
\text { overall } \mathbf{N}\end{array}$ & $\begin{array}{l}\text { Incident } \\
\text { non-dipping } \\
\mathbf{N}(\%)\end{array}$ & $\begin{array}{l}\text { Adjusted } \\
\text { relative risk } \\
(95 \% \mathrm{Cl})\end{array}$ \\
\hline \multicolumn{2}{l}{$\begin{array}{l}\text { Systolic non-dipping } \\
(\mathrm{n}=199)\end{array}$} & & \\
$<1$ & 73 & $8(11)$ & 1.0 (reference) \\
$1-4.9$ & 60 & $8(13)$ & $1.27(0.52$ to 3.09$)$ \\
$5-14.9$ & 38 & $8(21)$ & $2.00(0.77$ to 5.19$)$ \\
$\geq 15$ & 28 & $10(36)$ & $2.84(1.10$ to 7.29$)$ \\
Diastolic non-dipping $(\mathrm{n}=215)$ & & 1.0 (reference) \\
$<1$ & 83 & $6(7)$ & $0.48(0.10$ to 2.39$)$ \\
$1-4.9$ & 61 & $2(3)$ & $1.62(0.46$ to 5.76$)$ \\
$5-14.9$ & 40 & $5(13)$ & $4.27(1.20$ to 15.13$)$ \\
$\geq 15$ & 31 & $7(23)$ & \\
\hline
\end{tabular}

${ }^{*}$ Among participants with baseline normal blood pressure dipping and no use of antihypertensive medication, followed over an average of 6.6 years. All models adjusted for age, sex, race, body mass index, waist-to-hip ratio, current smoking alcohol consumption, and NREM AHI. P-trend for incident systolic and diastolic non-dipping with increasing REM AHI categories were 0.021 and 0.024 , respectively. AHI, apnoea hypopnoea index; NREM, non-rapid eye movement; REM, rapid eye movement.

or several variables from the baseline polysomnogram, including sleep efficiency, WASO and minutes of slow wave sleep (stage NREM 3), did not substantially change the relationship between and did not change the statistical significance of the AHI categories (REM, NREM and total AHI) and incident non-dipping (data not shown). We did not find any significant interaction between REM AHI categories and reported sleepiness (interaction coefficient $p$ values $=0.53$ and $p=0.75$ for systolic and diastolic non-dipping outcomes, respectively) or baseline hypertension status $(\mathrm{p}=0.38$ and $\mathrm{p}=0.65$ for systolic and diastolic, respectively).

\section{DISCUSSION}

In this longitudinal analysis of a population-based sample, we found that OSA in REM sleep, independent of NREM OSA, is associated with the development of nocturnal systolic and diastolic non-dipping of BP over an average follow-up period of 6.6 years among participants who initially were normal nocturnal BP dippers. The association showed a dose-response gradient: individuals with a baseline REM AHI $\geq 15$ had an approximately threefold greater relative risk of developing systolic non-dipping and a fourfold greater relative risk of developing diastolic non-dipping compared with those with REM $\mathrm{AHI}<1$. To our knowledge, this is the first study to evaluate the association of REM-related OSA and non-dipping in a sample selected without regard for the presence of OSA symptoms or other related morbidities. These unique findings suggest a role for OSA in REM sleep in the development of non-dipping of nocturnal BP.

The current study demonstrates that REM AHI, but not NREM or total AHI, is significantly associated with nocturnal diastolic BP dipping. Although several cross-sectional studies have reported a higher prevalence of non-dipping in moderate to severe OSA, ${ }^{12}$ only one longitudinal population-based study has reported an association between OSA and incident non-dipping of systolic BP. ${ }^{11}$ The direction of causality is further strengthened by studies that have shown CPAP therapy to lower night-time $\mathrm{BP}$ more than daytime $\mathrm{BP}^{13}{ }^{25-27}$ particularly in patients with resistant hypertension who are adherent to CPAP therapy. ${ }^{28} 29$ We previously reported that in the Wisconsin Sleep Cohort, total AHI severity was associated with nocturnal systolic, but not diastolic, dipping; however, that study did not attempt to differentiate between REM and NREM AHI. ${ }^{11}$ Accordingly, our current findings of a longitudinal association between REM AHI, but not NREM AHI, and diastolic nondipping provides novel insight regarding the potential impact of REM OSA on diastolic BP, an important predictor of cardiovascular risk in middle age. ${ }^{30}$ This is highly relevant given the mean age of our subjects (ie, $\leq 50$ years).

Findings from the present study as well as prior studies linking OSA to non-dipping are clinically relevant because of established associations between non-dipping and poor cardiovascular prognosis. ${ }^{5}$ OSA has been independently associated with cardiovascular morbidity and mortality and non-dipping may be one of several possible mechanisms by which such outcomes may be initiated or exacerbated in OSA. ${ }^{8-10}$ The acute and recurrent episodes of complete (apnoea) or partial (hypopnoea) obstruction of the upper airway in patients with OSA can lead to significant swings in intrathoracic pressure, intermittent hypoxaemia and hypercapnia, cortical microarousals, increased oxidative stress, and sleep fragmentation. Moreover, apnoeas and hypopneas cause temporary and significant elevations of BP (ie, up to $30-40 \mathrm{~mm} \mathrm{Hg}$ lasting $20-30 \mathrm{~s}$ ), likely via pathways initiated by sympathetic activation. ${ }^{13}$ It is possible that these repeated bouts of sympathetic activation prevent complete normalisation of BP in the time between apnoeas and hypopneas, preventing the normal BP dip from occurring.

REM OSA may have a greater impact on sympathetic activation and non-dipping of nocturnal BP than NREM OSA. Using beat-to-beat BP measurements and recordings of sympathetic nerve activity via microneurography in patients with OSA, Somers et $a l^{13}$ found an increase in mean BP from 92 $\pm 4.5 \mathrm{~mm} \mathrm{Hg}$ during quiet wakefulness to $116 \pm 5 \mathrm{~mm} \mathrm{Hg}$ in NREM sleep and $127 \pm 7 \mathrm{~mm} \mathrm{Hg}$ during REM sleep. Moreover, sympathetic activity was highest during REM sleep. In another study of 16 patients with OSA and untreated hypertension, beat-to-beat BP measured invasively revealed a higher systolic BP in REM sleep compared with NREM sleep $(148 \pm 29 \mathrm{~mm} \mathrm{Hg}$ vs $134 \pm 24 \mathrm{~mm} \mathrm{Hg}$, respectively). ${ }^{31}$ The higher BP seen during REM sleep in individuals with OSA may be related to the fact that, compared with events in NREM, obstructive apnoeas and hypopneas during REM sleep last longer and are associated with significantly larger degrees of oxygen desaturation. ${ }^{16} 17$ Moreover, REM sleep is associated with increased sympathetic activation and reduced vagal tone compared with NREM sleep in normal subjects and even more so in patients with OSA. ${ }^{13} 15$ Therefore, obstructive respiratory events in REM sleep, a state of sleep that is already under increased sympathetic dominance, could increase the risk for non-dipping compared with NREM sleep.

Our findings may have important clinical implications for the duration of CPAP use that is needed to reverse or decrease the risk of nocturnal BP non-dipping. In a recent randomised controlled trial of patients with OSA and resistant hypertension, there was a significant positive correlation between hours of CPAP use and the decrease in $24 \mathrm{~h}$ mean and nocturnal $\mathrm{BP}^{32}$ This positive correlation between hours of CPAP use and the decrease in $24 \mathrm{~h} \mathrm{BP}$ was also observed in a meta-analysis. ${ }^{26}$ Grimaldi $e{ } a l^{33}$ reported that 3 and 4 h of CPAP use after lights are turned off would leave the vast majority of obstructive events during REM sleep untreated while $7 \mathrm{~h}$ of CPAP therapy would cover most of REM sleep. Indeed, it is plausible that 
A

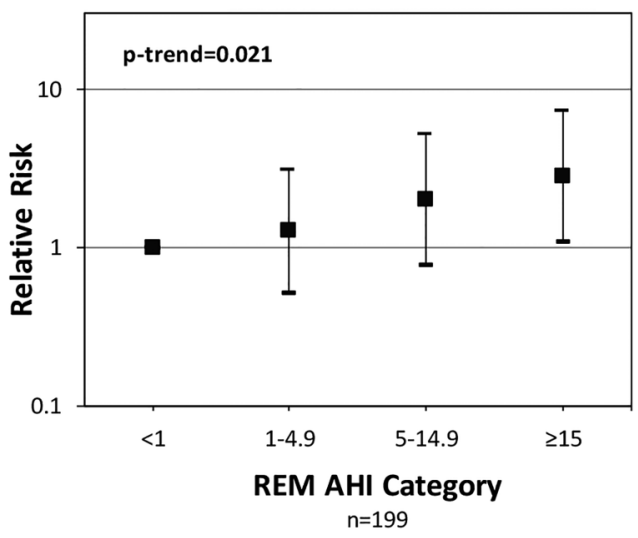

C

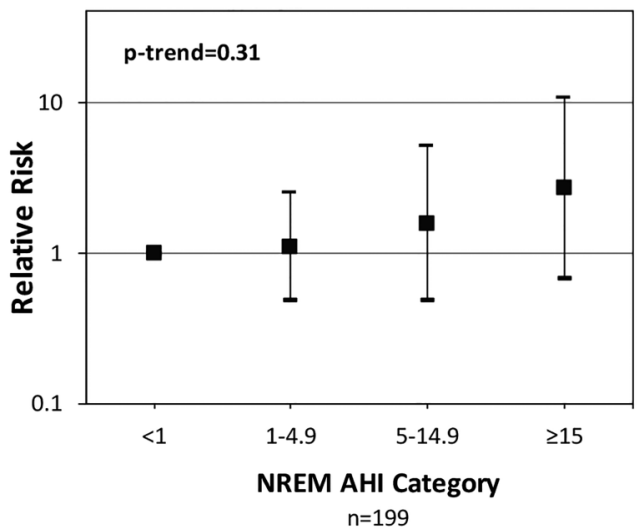

E

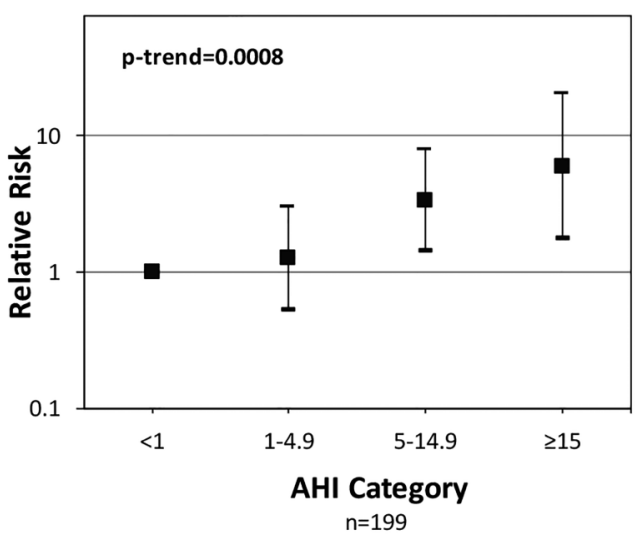

B

Diastolic nondipping

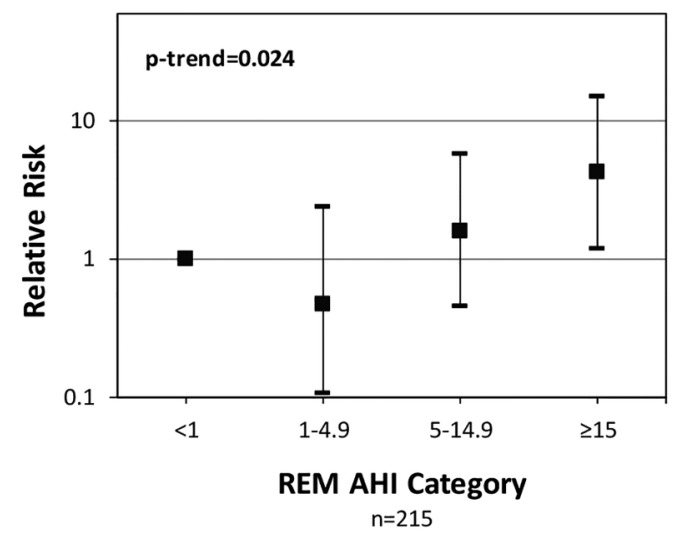

D

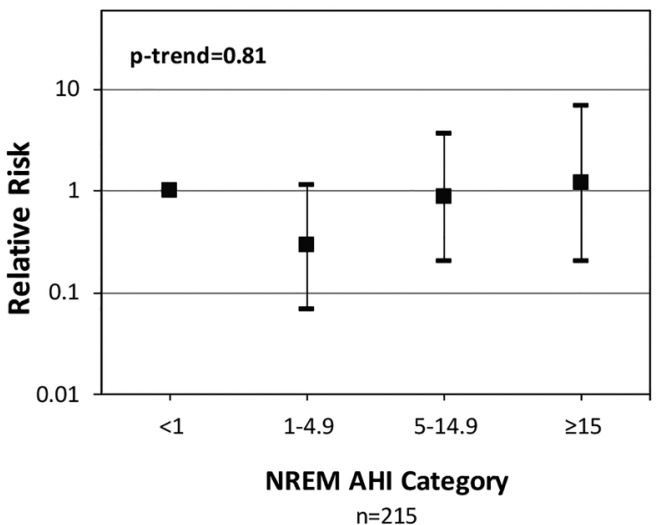

$\mathrm{F}$

Diastolic nondipping

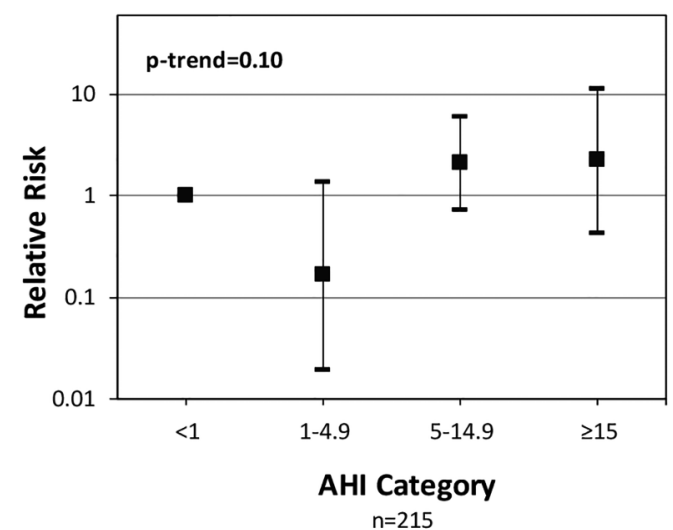

Figure 2 Relative risks and 95\% Cls from modified Poisson regression models quantifying the independent association between rapid eye movement (REM) apnoea hypopnoea index (AHI) severity categories and incident systolic and diastolic non-dipping (panels A and B) over an average follow-up of 6.6 years. Models are adjusted for age, sex, race, body mass index, waist-to-hip ratio, current smoking, alcohol consumption, and $\log _{2}$ (non-rapid eye movement (NREM) AHI+1) which allows for the coefficients to be interpreted as the 'effect' of a twofold increase in NREM AHI (1 was added to NREM AHI in the argument of the logarithm to allow for analysis of zero values). NREM AHI was not significant in these two models. Panels C and D explore the association between NREM AHI severity categories and incident non-dipping adjusted for age, sex, race, body mass index, waist-to-hip ratio, current smoking, alcohol consumption, and $\log _{2}(\mathrm{REM} A H I+1)$. NREM AHI categories were not associated with incident systolic or diastolic non-dipping. In contrast, doubling of REM AHI had a relative risk of 1.37 (95\% Cl 1.13 to $1.65 ; \mathrm{p}=0.0011)$ for incident systolic non-dipping and $1.50(95 \% \mathrm{Cl} 1.17$ to $1.93 ; p=0.0015)$ for incident diastolic non-dipping. Panels $\mathrm{E}$ and $\mathrm{F}$ illustrate the association between incident non-dipping and total AHI categories adjusted for age, sex, race, body mass index, waist-to-hip ratio, current smoking, and alcohol consumption. Increasing total AHI categories was independently associated with incident systolic non-dipping, but not diastolic.

reduced CPAP adherence and the predominantly untreated OSA during REM sleep (which prevails during the latter hours of normal nocturnal sleep) may explain the negative or modest effects of CPAP therapy on BP control in randomised clinical trials. Further research is needed to establish the optimal duration of CPAP use to decrease the risk of developing nondipping and to reverse an already established non-dipping pattern in patients with OSA. Moreover, non-intrusive 
technologies enabling frequent and accurate assessments of vascular function during different stages of sleep can significantly enhance our understanding of the impact of OSA in REM vs NREM sleep.

Our study has some limitations. First, even though we included NREM AHI as a covariate in our models, there may be residual confounding effects of OSA events not occurring during REM sleep. However, we have previously reported a persistent association between REM AHI and hypertension after excluding subjects with NREM AHI $>5 .{ }^{18}$ Second, in our study hypopneas were classified using $\geq 4 \%$ oxygen desaturation. We do not have data on REM and NREM microarousals for a substantial portion of the cohort. We therefore cannot ascertain whether the observed adverse effect of REM OSA on nondipping would still be detected using hypopnoea definitions incorporating $\geq 3 \%$ oxygen desaturation or microarousals. Third, oxygen saturation metrics such as oxygen desaturation index and per cent of sleep time below 90\% oxygen saturation were not available for the majority of polysomnograms prior to the year 2000, when fully digitalised data collection was initiated in the WSCS. Therefore, for the present analyses, we rely only on AHI as the metric of OSA severity. Fourth, our findings may not be generalisable to African Americans or Hispanics since $98 \%$ of the subjects included in our cohort were non-Hispanic whites. Lastly, our REM AHI categories were arbitrary and similar to cutoffs used in clinical practice. However, models that used REM AHI categories based on REM AHI quartiles $(<0-0.6,0.6-2.9,3.0-12.2,>12.2)$ led to very similar results.

Our study has several noteworthy strengths. First, we believe that our conservative inclusion criterion of at least $30 \mathrm{~min}$ of REM sleep reduces the possibility of exaggerating the effects of REM OSA in individuals with short REM duration. Second, we used actual sleep and wake times recorded by participants and not arbitrary preset times during the $24 \mathrm{~h}$ ABPM. This approach provides additional accuracy in defining sleep and wake BP. Third, ABPM was performed at home and not on the same night of in-laboratory polysomnography, thus eliminating the possibility of artificially induced reduction of sleep efficiency and quality related to the discomfort of ABPM measuring BP every $30 \mathrm{~min}$. Fourth, our study was performed in a populationbased working cohort, enabling us to prospectively determine the association of REM OSA and non-dipping in a non-clinical population with occult or undiagnosed OSA. Fifth, with our sample size we were able to account for the confounding effects of several important covariates on the relationship of REM OSA and non-dipping. Lastly, our longitudinal analytic approach has the advantage of having the correct temporal sequence and therefore strengthens the evidence that OSA in REM sleep may have a causal role in the development of nocturnal non-dipping of BP.

In summary, our findings of a strong longitudinal association of REM OSA with nocturnal non-dipping of BP has clinical and public health relevance. Additional studies are needed to establish mechanisms and pathways by which events during REM sleep lead to increased risk of nocturnal non-dipping of BP and to explore whether effective treatment that covers most of REM sleep can reduce the risk of non-dipping.

\footnotetext{
Author affiliations

${ }^{1}$ Department of Medicine, Section of Pulmonary and Critical Care, Sleep Disorders Center, University of Chicago, Chicago, Illinois, USA

2Department of Population Health Sciences, School of Medicine and Public Health, University of Wisconsin-Madison, Madison, Wisconsin, USA

${ }^{3}$ Department of Medicine, School of Medicine and Public Health, University of
}

Wisconsin-Madison, Madison, Wisconsin, USA

${ }^{4}$ Department of Kinesiology and Integrative Physiology, Michigan Technological University, Houghton, Michigan, USA

Acknowledgements The authors thank Terry Young, PhD, Mari Palta, PhD, Amanda Rasmuson, MS, Kathryn Pluff, Katherine Stanback, Robin Stubbs, Jodi Barnet, MS, Mary Sundstrom, Steven Barczi, MD, and Mihai Teodorescu, MD for their contributions to study implementation and data collection.

Contributors BM, LAF and PEP designed the study and/or the analysis. BM, EWH, LAF and PEP performed all analyses. PEP obtained study funding. PEP and KMH oversaw data collection. BM, LAF, EWH, KMH, JRC, and PEP participated in data interpretation and manuscript preparation. BM and PEP oversaw all analyses and take full responsibility for the work as a whole, including the study design, access to data, and the decision to submit and publish the manuscript. All authors approved this manuscript in its final form.

Funding Supported by National Institutes of Health grants R01HL062252 and 1UL1RR025011. BM is supported by R01HL119161. JRC is supported by R15HL122919.

Competing interests BM has served as a consultant to Philips/Respironics and has received research support from Philips/Respironics. The other authors do not have any competing interests to report.

Ethics approval University of Wisconsin Institutional Review Board.

Provenance and peer review Not commissioned; externally peer reviewed.

\section{REFERENCES}

1 Pickering TG, White WB, Giles TD, et al. When and how to use self (home) and ambulatory blood pressure monitoring. J Am Soc Hypertens 2010;4:56-61.

2 Pickering TG, Shimbo D, Haas D. Ambulatory blood-pressure monitoring. N Engl J Med 2006;354:2368-74.

3 Dolan E, Stanton A, Thijs L, et al. Superiority of ambulatory over clinic blood pressure measurement in predicting mortality: the Dublin outcome study. Hypertension 2005;46:156-61.

4 Chobanian AV, Bakris GL, Black HR, et al. The Seventh Report of the Joint National Committee on Prevention, Detection, Evaluation, and Treatment of High Blood Pressure: the JNC 7 report. JAMA 2003;289:2560-72.

5 Staessen JA, Thijs L, Fagard R, et al. Predicting cardiovascular risk using conventional vs ambulatory blood pressure in older patients with systolic hypertension. Systolic Hypertension in Europe Trial Investigators. JAMA 1999;282:539-46.

6 Boggia J, Li Y, Thijs L, et al. Prognostic accuracy of day versus night ambulatory blood pressure: a cohort study. Lancet 2007;370:1219-29.

7 Peppard PE, Young T, Barnet JH, et al. Increased prevalence of sleep-disordered breathing in adults. Am J Epidemiol 2013;177:1006-14.

8 Peppard PE, Young T, Palta M, et al. Prospective study of the association between sleep-disordered breathing and hypertension. N Engl J Med 2000;342:1378-84.

9 Marin JM, Agusti A, Villar I, et al. Association between treated and untreated obstructive sleep apnea and risk of hypertension. JAMA 2012;307:2169-76.

10 Campos-Rodriguez F, Martinez-Garcia MA, de la Cruz-Moron I, et al. Cardiovascular mortality in women with obstructive sleep apnea with or without continuous positive airway pressure treatment: a cohort study. Ann Intern Med 2012;156:115-22.

11 Hla KM, Young T, Finn L, et al. Longitudinal association of sleep-disordered breathing and nondipping of nocturnal blood pressure in the Wisconsin Sleep Cohort Study. Sleep 2008;31:795-800.

12 Seif F, Patel SR, Walia HK, et al. Obstructive sleep apnea and diurnal nondipping hemodynamic indices in patients at increased cardiovascular risk. J Hypertens 2014;32:267-75.

13 Somers VK, Dyken ME, Clary MP, et al. Sympathetic neural mechanisms in obstructive sleep apnea. J Clin Invest 1995;96:1897-904.

14 Kohler M, Stradling JR. CrossTalk proposal: Most of the cardiovascular consequences of OSA are due to increased sympathetic activity. J Physiol 2012;590:2813-2815; discussion 2823.

15 Somers VK, Dyken ME, Mark AL, et al. Sympathetic-nerve activity during sleep in normal subjects. N Engl I Med 1993;328:303-7.

16 Peppard PE, Ward NR, Morrell MJ. The impact of obesity on oxygen desaturation during sleep-disordered breathing. Am J Respir Crit Care Med 2009;180:788-93.

17 Krieger J, Sforza E, Boudewijns A, et al. Respiratory effort during obstructive sleep apnea: role of age and sleep state. Chest 1997;112:875-84.

18 Mokhlesi B, Finn LA, Hagen EW, et al. Obstructive sleep apnea during REM sleep and hypertension. results of the Wisconsin Sleep Cohort. Am J Respir Crit Care Med 2014; 190:1158-67.

19 Chami HA, Baldwin CM, Silverman A, et al. Sleepiness, quality of life, and sleep maintenance in REM versus non-REM sleep-disordered breathing. Am J Respir Crit Care Med 2010;181:997-1002. 
20 Mokhlesi B, Punjabi NM. 'REM-related' obstructive sleep apnea: an epiphenomenon or a clinically important entity? Sleep 2012;35:5-7.

21 Young T, Palta M, Dempsey J, et al. The occurrence of sleep-disordered breathing among middle-aged adults. N Engl I Med 1993;328:1230-5.

22 Hla KM, Young TB, Bidwell T, et al. Sleep apnea and hypertension. A population-based study. Ann Intern Med 1994;120:382-8.

23 Zou G. A modified Poisson regression approach to prospective studies with binary data. Am J Epidemiol 2004;159:702-6.

24 Pankow W, Nabe B, Lies A, et al. Influence of sleep apnea on 24-hour blood pressure. Chest 1997:112:1253-8.

25 Hla KM, Skatrud JB, Finn L, et al. The effect of correction of sleep-disordered breathing on BP in untreated hypertension. Chest 2002;122:1125-32.

26 Haentjens P, Van Meerhaeghe A, Moscariello A, et al. The impact of continuous positive airway pressure on blood pressure in patients with obstructive sleep apnea syndrome: evidence from a meta-analysis of placebo-controlled randomized trials. Arch Intern Med 2007;167:757-64.

27 Hu X, Fan J, Chen S, et al. The role of continuous positive airway pressure in blood pressure control for patients with obstructive sleep apnea and hypertension: a meta-analysis of randomized controlled trials. J Clin Hypertens (Greenwich) 2015;17:215-22.
28 Barbe F, Duran-Cantolla J, Sanchez-de-la-Torre M, et al. Effect of continuous positive airway pressure on the incidence of hypertension and cardiovascular events in nonsleepy patients with obstructive sleep apnea: a randomized controlled trial. JAMA 2012;307:2161-8.

29 Lloberes P, Sampol G, Espinel E, et al. A randomized controlled study of CPAP effect on plasma aldosterone concentration in patients with resistant hypertension and obstructive sleep apnea. J Hypertens 2014;32:1650-1657; discussion 1657.

30 Franklin SS, Larson MG, Khan SA, et al. Does the relation of blood pressure to coronary heart disease risk change with aging? The Framingham Heart Study. Circulation 2001;103:1245-9.

31 Peter JH, Grote L, Fus E, et al. REM-sleep-hypertension in obstructive sleep apnea. Eur J Med Res 1995;1:132-6.

32 Martinez-Garcia MA, Capote F, Campos-Rodriguez F, et al. Effect of CPAP on blood pressure in patients with obstructive sleep apnea and resistant hypertension: the HIPARCO randomized clinical trial. JAMA 2013;310:2407-15.

33 Grimaldi D, Beccuti G, Touma C, et al. Association of obstructive sleep apnea in rapid eye movement sleep with reduced glycemic control in type 2 diabetes: therapeutic implications. Diabetes Care 2014;37:355-63. 